\title{
Automatic Generation of Update Rules to Enforce Consistency Constraints in Design Databases
}

\author{
James Brawner \\ St. John's University \\ Jamaica, NY 11439 \\ Joan Peckham \\ Univ. of Rhode Island \\ Kingston, RI 02881
}

\author{
Bonnie MacKellar \\ Western Connecticut State Univ. \\ Danbury, CT 06268 \\ James Vorbach \\ St. John's University \\ Jamaica, NY 11439
}

\begin{abstract}
Database systems for computer-aided design (CAD) are characterised by structural complexity and nonstandard relationship types. Integrity constraints typically describe the semantics of these relationships. Enforcing such constraints in the face of user-initiated changes to the database is necessary to ensure that the database is consistent and the corresponding design is valid. Typically, update rules are specified to maintain integrity constraints. In this paper, we concentrate on the automatic generation of update rules from integrity constraints. Our methodology generates two types of update rules, which are guaranteed to result in a consistent database state. The database designer is provided with the flexibility of alternative semantics as a result of the two update rule types. Tests for integrity violations and repair actions are both generated during a single analysis. The underlying formalism expresses constraints, updates, and rules within the same language; thus the same proof theory applies to all components. This simplifies the overall analysis and enables concise correctness proofs.
\end{abstract}

\section{INTRODUCTION}

Database systems for computer-aided design (CAD) are characterised by structural complexity and nonstandard relationship types. Structural complexity occurs because a typical CAD object is made up of many sub-objects. Much of the meaning of a CAD object is based on relationships among its component objects. In particular, the behavior of the object is often determined by the kind of relationships among its component objects. For example, consider a design for a wall containing a window assembly. The wall contains 
a hole for the window, the window void, which in turn contains the window assembly. The window assembly itself is made up of a frame and at least one pane of glass. If the window void containing the window assembly is deleted, the window assembly must be moved; it is physically impossible for it to be contained by the wall at this point. If, on the other hand, only the window frame is moved, the object representing the window assembly must be deleted, even though the frame, pane and window void objects remain behind. This is because a window assembly object represents an aggregation of component objects which fit together in a particular way; once the physical connection is broken, the assembly no longer exists.

Integrity constraints typically describe the semantics of these relationships. Enforcing such constraints in the face of user-initiated changes to the database is necessary to ensure that the database is consistent and the corresponding design is valid. Integrity maintenance rules, which can be specified when the database schema is designed, can be used to enforce the integrity constraints. Unfortunately, such rules are difficult to correctly specify.

The synthesis of integrity maintenance rules can be viewed as a sequential two-phase process. The first phase, rule generation, has as input the set of integrity constraints and outputs candidate rules to enforce them. The second phase, rule analysis, determines rule behavioral properties, such as termination, uniqueness of the final state (confluence), and whether or not their execution will have a unique sequence of observable actions (determinism). The results of the rule analysis phase may be used as criteria for selecting from the candidate rules, which were produced in the rule generation phase.

The focus of this paper is on the process of rule generation. In our view, any approach towards rule generation should satisfy the following criteria: (1) The database designer should have a choice in rule execution semantics. For example, a given constraint can often be satisfied either by denying an update that would result in an inconsistent database state, or by allowing the update and executing corrective actions to restore consistency. (2) Update rules should be generated automatically from integrity constraints and should be provably correct. (3) The updates that may violate a constraint (threatening updates, in this work) and repair actions which assist in restoring a consistent state (assisting updates) should be automatically identified. (4) This analysis should be done at compile time, to the extent possible. In this paper we report on our efforts to develop a rule generation methodology. This methodology satisfies these criteria and permits a simpler and more integrated approach to rule generation than in related literature (Ceri et al. 1994, Ceri et al. 1990, Qian 1990, Urban et al. 1990, Urban et al. 1992, Urban et al. 1992).

We previously introduced a formal system called Event-Formula Logic (EFL) (Brawner et al. 1998) for characterising correctness of database updates. This earlier work was based on algorithmic logic (Mirk et al. 1986), a formal system for analysing iterative programs. Here we apply EFL to the problem of updating design databases. We introduce a rule methodology in 
which the database designer can choose among alternative update semantics formulated in two rule types: a Condition/Action (CA) rule and an Action/Condition/Correction (ACC) rule. EFL expresses constraints, updates, and rules within the same language; thus the same proof theory applies to all components. This simplifies the overall analysis and enables concise correctness proofs. Update rules, threatening and assisting updates are automatically derived through efficient syntactic methods and are provably correct.

Most work in the area of rule generation represents integrity constraints using some variant of FOL because of its unambiguous declarative semantics (Nicolas et al. 1978). Typically, an operational semantics is used for update rules for ease of implementation. The problem with this is that it is difficult to prove that the method of generating update rules from integrity constraints is correct. Having the same formal semantics for constraints and update rules simplifies the correctness proof of the algorithm for generating update rules. This is a particularly distinguishing contribution of our work.

\subsection{Related Work}

Related work in the area of rule generation includes CONTEXT (Urban et al. 1990, Urban et al. 1992, Urban et al. 1992) and the work of Ceri and Widom (Ceri et al. 1990, Ceri et al. 1994). In CONTEXT, constraints in first-order logic (FOL) are transformed into Horn clauses. A complex constraint can map to several Horn clauses; consequently, variables bound in the FOL constraint that are mapped into more than one Horn clause are no longer related. The constraint graphs, in addition to facilitating an effective analysis algorithm, tie together variables appearing in Horn clauses generated from the same FOL constraint. The analysis identifies threatening operations and automatically generates repair actions as components of Integrity Maintenance Production Rules (IMPRs). In contrast, integrity constraints in this paper are analysed in their normalised FOL representation, eliminating transformations and thus simplifying both the analysis and the correctness proofs. In the work of Ceri et al. (Ceri et al. 1994, Ceri et al. 1990), rules are automatically generated from constraints using the concept of invalidating operations, which are those operations which can result in the violation of an integrity constraint. Invalidating operations, which are determined based on syntactic analysis, form the triggers of integrity enforcing rules. In our work, we introduce a formal basis for proving the correctness of threatening updates, which are equivalent to invalidating operations, and a more concise syntactic method for their derivation. In addition, we introduce the dual notion of assisting updates, which are candidate corrective actions generated simultaneously with the threatening updates.

Qian introduces a unified framework in the area of transaction synthesis (Qian 1990). Here, a first-order situational logic-based method is introduced 
for deriving transactions from specifications. The transactions generated are guaranteed to preserve the validity of integrity constraints. This approach, however, is too restrictive in applications where it is appropriate to allow temporarily inconsistent database states with a promise of later restoration to consistency via propagated repair actions.

Schewe et al. (Schewe et al. 1992) introduce an approach to the integrity enforcement problem in object-oriented databases. For each class in the schema, the methods are automatically augmented to enforce the schema constraints. The constructed method is minimal with respect to the degree by which the given method is augmented. An analogous but model independent result is obtained in our paper by applying the notion of the weakest precondition (Mirk et al. 1986) as the formal machinery underlying the rule generator. Wüthrich (Wüthrich 1993) introduces an efficient method for enforcing constraints in knowledge bases. An update, which includes any necessary repair actions, is derived from a given update and the constraints. The solution is minimal though not unique. Generating all minimal solutions is avoided by requiring user interaction when the next step in the derivation is non-deterministic. Fraternali (Fraternali et al. 1993) provides a concise survey of related literature.

The rule generation methodology introduced in this paper has four principal advantages. First, it provides a unifying formal framework for specifying constraints, operations, and rules. This framework simplifies the proving of important meta-properties, such as soundness and completeness. A soundness result for derived rules is given. While in general, completeness escapes formalisation due to the designer interaction that may be necessary to arbitrate among alternative corrective actions, with some simplifying assumptions, a completeness result is also obtained. Second, this approach gives us a purely syntactic method for identifying invalidating operations. Third, the approach generalises easily towards analysing multiple constraints. As in (Qian 1990), multiple constraints can be conjuncted and then analysed. Lastly, the rules are easily transformed into production rules or ECA rules, which are languages typically used by current methods for the second phase, rule analysis (Widom et al. 1992, Ceri et al.`1994, Ceri et al. 1990, Urban et al. 1992).

\section{PROBLEM DOMAIN}

Our example domain is a CAD database that stores information about building plans. Such a database would be manipulated by architects during the course of designing a building. The details of this system have been reported in (Peckham et al. 1995). In particular, its data model is based on both objects and relationships. We consider the problem of moving the components of a window assembly for illustration. The components of a window assembly may be seen in Figure 1.

This operation affects the various part relationships which model this example, which can be seen in Figure 2. A window is modeled by a window 


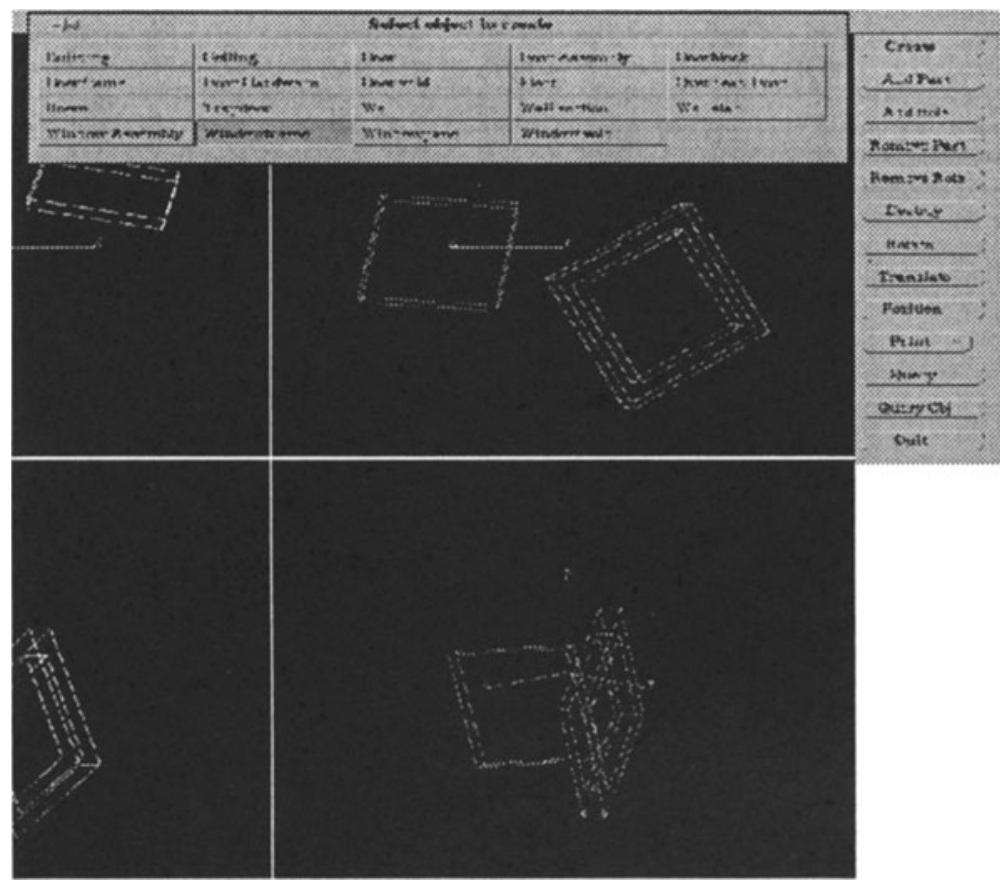

Figure 1 Components of a simple window assembly

assembly which is composed of a window frame and its related glass pane(s). Positional dependencies exist among the objects. A frame must "fit" in the window assembly. When a frame is moved its position is re-calculated. If its new position fails to satisfy the position dependency with its window assembly, then that part relationship is updated. At schema design time the specification of these integrity constraints serves as input to the process of deriving integrity maintenance rules.

Each object instance contains both symbolic and geometric information. For example, a door's color is part of its symbolic aspect, whereas its shape and position are part of its geometric aspect. There are many types of assemblies in a building in which the components are of predetermined types and fit together in a predictable way. Door and window assemblies are examples. In the current prototype, a window assembly is modeled fairly simplistically, consisting only of a frame and pane of glass, as shown in Figure 1. In turn, window assemblies fit into window voids.

Each type of object which could participate in an assembly carries with it a set of default "mate points" - points on the solid which would normally connect to points on other objects in an assembly. For example, on a pane of glass, these points would be defined as default mate points as in Figure 3. Knowledge of how to fit two objects together in an assembly can be expressed as mating constraints, which are a way of specifying contact between points, lines, or 
surfaces on solids to be assembled (Mullins et al. 1993). Mating constraints can be associated with either object or relationship instances. For example, the constraint specifying how to fit the components of a window assembly together is associated with the window assembly class, while knowledge of how to fit a window void into a wall rests with the part relationship type defined between walls and window voids.

In the case of three-point coincidence, the assembly semantics might be determined by these mating constraints

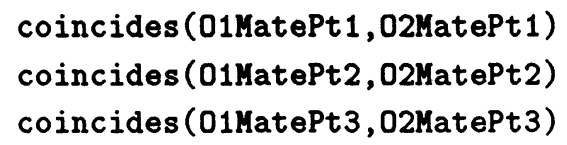

Therefore, if the WindowAssembly is moved and the mating constraints no longer hold, then the part relationship must be broken and possibly recreated. Currently, we only support simple point coincidence, but this approach can be extended to other kinds of mating constraints as in (Kramer 1991).

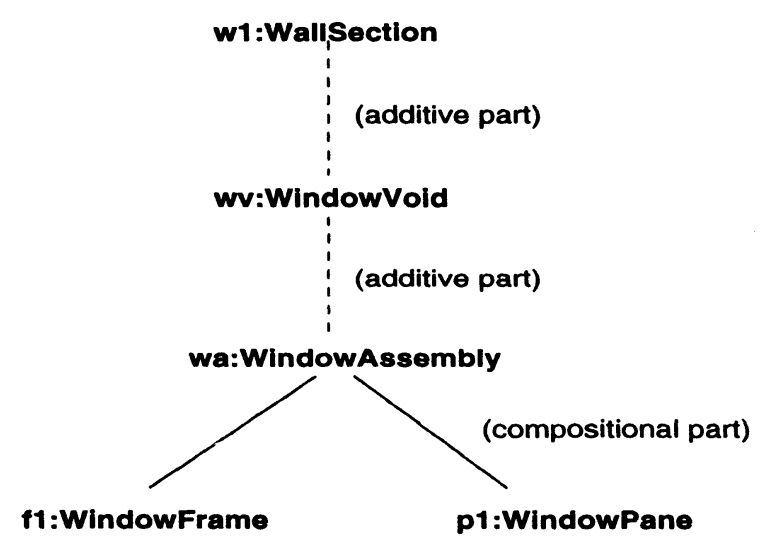

Figure 2 Relationship diagram for a wall section and window assembly

\subsection{Formal Representation}

A database designer constructs a conceptual specification to define the set of consistent states of the database. The specification restricts the states to those which accurately model the application. The specification can be viewed as a theory with its semantics given by the underlying database. The EFL language (Brawner et al. 1998) formally expresses conceptual specifications and is characterised by a model-theoretic semantics. The key point in expressing the properties of database updates is the dynamic formula $M \alpha$, where $M$ 
is an event and $\alpha$ is a formula. Of particular interest are the atomic events corresponding to the insertion and deletion of objects and relationships, and the rule events. The meaning of an event is given by the assignment of a binary relation over database states. The binary relation consists of the set of all pairs of possible states before and after the execution of the event. In contrast, the meaning of a static formula, $\alpha$, is given by its satisfaction with respect to a particular database state and a valuation; i.e. an assignment of constants to the variables in $\alpha$. We restrict our attention here to integrity constraints modeled as static formulas. The dynamic formula $M \alpha$ is satisfied in the current database state if $\alpha$ is satisfied in the database state reached after $M$ executes. For example, the dynamic formula $\langle+\tau\rangle \alpha$ is satisfied in a database state $I$ if and only if the formula $\alpha$ is satisfied in the database state $J$ that results from inserting the atomic formula $\tau$ into the state $I$. The reader is referred to the appendix for the formal definition of the language and semantics of EFL.

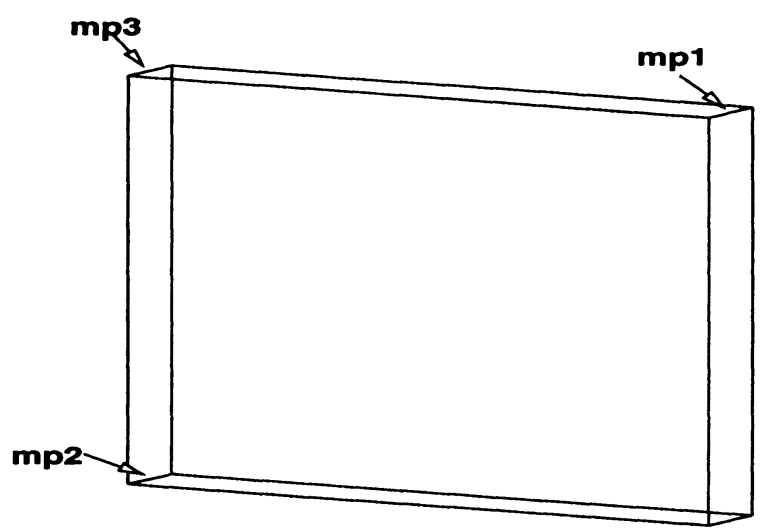

Figure 3 A pane and default mate points

\subsection{Examples}

We give the following constraints and corresponding update rules to use for illustration in the discussion that follows.

Constraint 1: A window assembly must have a frame.

WindowAssemb $(W A) \rightarrow \exists F(F r a m e(F) \wedge$ has_part $(W A, F))$

Update Rule: If a has_part relationship is deleted then the corresponding WindowAssemb object is also deleted. 


$$
\begin{aligned}
& <- \text { has_part }(W A, F)> \\
& <\text { if }(F r a m e(F) \wedge W \text { indowAssemb }(W A)) \text { then } \\
& \quad<-W \text { indowAssemb }(W A)> \\
& \text { fi }>
\end{aligned}
$$

Constraint 2: The frame and pane mate points must coincide.

$($ WindowAssemb $(W A) \wedge$ Frame $(F) \wedge \operatorname{Pane}(P)$

$\wedge h a s_{-} p a r t(W A, F) \wedge$ has_part $\left.(W A, P)\right) \rightarrow$

$\exists x, y($ MatePoints $(F, x) \wedge M$ atePoints $(P, y) \wedge$ coincides $(x, y))$

where coincides is interpreted as a Boolean function.

An object's mate points are dependent on its position in the world coordinate system, or on its transformation matrix. We can incorporate this dependence into our system using a function (or method) $\phi$, which assigns to each transformation matrix $T M$ and Frame $\mathrm{F}$, an ordered set of world mate points $M P_{W}$. The positional dependency can then be modeled by the following

Constraint 3:

$$
\text { Position }(F, T M) \wedge M \text { atePoints }\left(F, M P_{W}\right) \rightarrow \phi(T M, F)=M P_{W}
$$

Then we can consider the operation $\langle\operatorname{move}(F, T M, T M 2)>$, defined as

$$
<-\operatorname{position}(F, T M)>;<+ \text { position }(F, T M 2)>
$$

\section{Update Rule:}

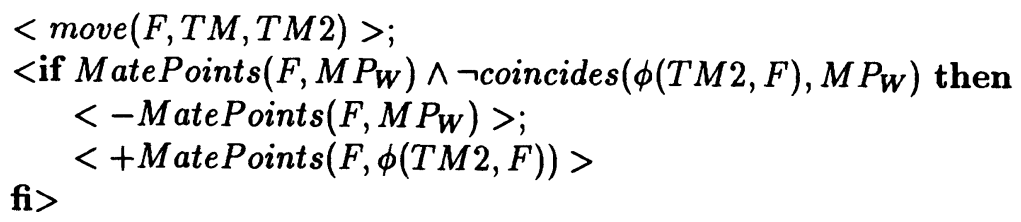

It is important to note that one update rule can trigger another, leading to propagations across the database. For example, the following rule chain can be derived.

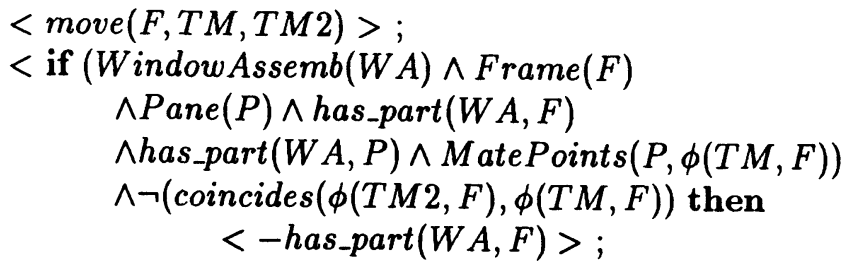


fi $>$

$$
<-W i n d o w A s s e m b(W A)>
$$

The rule chain is constructed by substituting integrity maintenance rules for the corresponding atomic updates in the action components of other rules. The atomic updates being replaced are the triggers for the rules that are replacing them. The replacement is consistent with the inductive definition of events in EFL and hence the result is guaranteed to be a syntactically correct rule.

\section{INTEGRITY CONSTRAINT ANALYSIS}

In order to derive update rules, some formal machinery is needed to relate updates and static formulas in such a way that the formula is guaranteed to hold after the update executes. This formalism facilitates an automatic procedure for deriving update rules; furthermore, the underlying logic allows us to prove the correctness of the derived update rules. The approach taken here is to apply the notion of the weakest precondition as the key component of the formalism. This section defines this notion and shows its equivalence to a syntactic variation of an integrity constraint with respect to a given update. This equivalence is the basis for the automatic derivation procedure.

\subsection{Preliminary Notions}

An integrity constraint is interpreted as a static first-order formula. As previously mentioned, a static formula is one without updates. Formulas are not restricted to be Horn clauses with the concomitant difficulties that would entail (Urban et al. 1990). Instead, our representation is the standard conjunctive normal form (CNF) in first-order predicate logic. For convenience, we recall the standard definition below, along with the notions of reduced $\mathrm{CNF}$ and ordering with respect to a specific atomic formula.

Let $P=\left\{P_{1}, \ldots, P_{n}\right\}$ be a finite set of distinct atomic formulas. A formula is in conjunctive normal form with respect to $P$ if and only if it has the form

$$
\left(P_{11} \vee \cdots \vee P_{1 n}\right) \wedge\left(P_{21} \vee \cdots \vee P_{2 n}\right) \wedge \cdots \wedge\left(P_{m 1} \vee \cdots \vee P_{m n}\right)
$$

where each $P_{i j}$ is either $P_{j}$ or $\neg P_{j}$. Furthermore, the conjunctive normal form is reduced if and only if there does not exist an equivalent formula in conjunctive normal form with respect to any proper subset of $P$. For example, the formula

$$
\left(P_{1} \vee P_{2}\right) \wedge\left(\neg P_{1} \vee P_{2}\right)
$$


is in conjunctive normal form with respect to $\left\{P_{1}, P_{2}\right\}$, but not reduced conjunctive normal form, since

$$
\left(P_{1} \vee P_{2}\right) \wedge\left(\neg P_{1} \vee P_{2}\right) \Leftrightarrow P_{2}
$$

and $P_{2}$ is in conjunctive normal form with respect to $\left\{P_{2}\right\} \subset\left\{P_{1}, P_{2}\right\}$. Finally, we say that the reduced conjunctive normal form is ordered with respect to an atomic formula $\tau$ if $\tau$ is the first atomic formula in each conjuncted clause and the conjuncted clauses are ordered so that the ones in which $\tau$ appears as a positive literal come first. In terms of the notation above, this means $\tau$ is $P_{1}$ and there exists an integer $k, 0 \leq k \leq m$, such that $P_{r 1}$ is $P_{1}$ for $1 \leq r \leq k$ and $\neg P_{1}$ for $k<r \leq m$.

\subsection{The Weakest Precondition}

After normalisation, a constraint is analysed with respect to a particular update $M$ to determine its weakest precondition (Mirk et al. 1986). The importance of the weakest precondition in our analysis cannot be overstated; it is a formula that can be tested in the current database state to determine whether a given update will result in an inconsistent database state. This notion is the basis for the conditions in the update rules described in section 5: the Condition/Action (CA) rule and the Action/Condition/Correction (ACC) rule. If the execution of a given update might result in an inconsistent database state, these update rules can either roll back the threatening update, or perform corrective action to restore consistency.

Definition 1 The weakest precondition of a formula $\alpha$ with respect to an event $M$ is a formula $\beta$ such that

1. $\beta \Rightarrow M \alpha$ (i.e. $\beta$ is a precondition); and

2. if $\gamma \Rightarrow M \alpha$ for some formula $\gamma$ then $\gamma \Rightarrow \beta$.

It is clear that $M \alpha$ itself is the weakest precondition since it trivially satisfies both conditions. We seek to determine an equivalent static formula with which we can test prior to execution whether the execution of an event will result in the violation of an integrity constraint. Our candidate for this weakest precondition in the case of atomic updates is the static formula defined below, which we term the discatenation of $\alpha$ with respect to $M$.

Definition 2 Let $M$ be an atomic update event $<+\tau>$ (respectively, $<$ $-\tau>)$ and $\alpha$ a static formula. The discatenation of $\alpha$ with respect to $M$ is a 
static formula $\beta$ such that

$$
\begin{array}{r}
\beta \wedge \tau \Leftrightarrow \alpha \wedge \tau \\
(\text { respectively, } \beta \wedge(\neg \tau) \Leftrightarrow \alpha \wedge(\neg \tau))
\end{array}
$$

and $\tau$ does not appear in the conjunctive normal form of $\beta$.

Notice that if $\tau$ does not appear in the conjunctive normal form of $\alpha$ then the discatenation of $\alpha$ with respect to both $\langle+\tau\rangle$ and $\langle-\tau\rangle$ is simply $\alpha$ itself.

Lemma 1 Let $\alpha$ be a static formula with distinct atomic formulas $\tau, P_{2}$, $P_{3}, \ldots, P_{n}$ and reduced conjunctive normal form ordered with respect to $\tau$ :

$$
\begin{array}{r}
\alpha \Leftrightarrow\left(\tau \vee P_{12} \vee \cdots \vee P_{1 n}\right) \wedge \cdots \wedge\left(\tau \vee P_{k 2} \vee \cdots \vee P_{k n}\right) \wedge \\
\left(\neg \tau \vee P_{(k+1) 2} \vee \cdots \vee P_{(k+1) n}\right) \wedge \cdots \wedge\left(\neg \tau \vee P_{m 2} \vee \cdots \vee P_{m n}\right)
\end{array}
$$

where each $P_{i j}$ is either $P_{j}$ or $\neg P_{j}$ for $i \geq 2$. Then

1. the discatenation $\beta$ of $\alpha$ with respect to $\langle+\tau\rangle$ is given by

$$
\beta \Leftrightarrow\left(P_{(k+1) 2} \vee \cdots \vee P_{(k+1) n}\right) \wedge \cdots \wedge\left(P_{m 2} \vee \cdots \vee P_{m n}\right) ; \text { and }
$$

2. the discatenation $\beta$ of $\alpha$ with respect to $\langle-\tau\rangle$ is given by

$$
\beta \Leftrightarrow\left(P_{12} \vee \cdots \vee P_{1 n}\right) \wedge \cdots \wedge\left(P_{k 2} \vee \cdots \vee P_{k n}\right) .
$$

Proof. From the laws of DeMorgan and distribution

$$
\begin{aligned}
\alpha \wedge \tau & \Leftrightarrow\left(P_{(k+1) 2} \vee \cdots \vee P_{(k+1) n}\right) \wedge \cdots \wedge\left(P_{m 2} \vee \cdots \vee P_{m n}\right) \wedge \tau \text { and } \\
\alpha \wedge(\neg \tau) & \Leftrightarrow\left(P_{12} \vee \cdots \vee P_{1 n}\right) \wedge \cdots \wedge\left(P_{k 2} \vee \cdots \vee P_{k n}\right) \wedge(\neg \tau)
\end{aligned}
$$

whence follow the formulas for $\beta$ given above.

Notice that the conjunctive normal form given above for $\beta$ may not be reduced. In general, we will express the discatenation in reduced conjunctive normal form; in that case, the form is unique up to rearrangement of literals.

The following theorem shows that the discatenation is indeed the weakest precondition with respect to atomic update events. The proof is given in the appendix.

Theorem 2 If $M$ is an atomic update event and $\alpha$ is any static formula then the weakest precondition of $\alpha$ with respect to $M$ is the discatenation $\beta$ of $\alpha$ with respect to $M$. 
Example 1 We use constraint 1 in the previous section for illustration.

$$
\alpha: W \operatorname{inAssemb}(W A) \rightarrow(\exists F(F r a m e(F) \wedge \text { has_part }(W A, F)))
$$

The Skolemised version is:

$$
\alpha^{*}: W \operatorname{inAssemb}(W A) \rightarrow\left(F r a m e(\sigma(W A)) \wedge h a s_{\text {_part }}(W A, \sigma(W A))\right)
$$

where $\sigma$ is the Skolem function replacing the existential operator. We use $W, f$, and $h$ as abbreviations for WinAssemb $(W A)$, Frame $(\sigma(W A))$, and has_part $(W A, \sigma(W A))$, respectively.

We give the reduced conjunctive normal form (CNF) representation of $\alpha^{*}$ with respect to each of these three atomic formulas, $W, f$, and $h$, and give the weakest preconditions for the corresponding atomic updates. Ordered with respect to $W, \alpha^{*}$ is equivalent to

$$
(\neg W \vee f \vee h) \wedge(\neg W \vee f \vee \neg h) \wedge(\neg W \vee \neg f \vee h)
$$

The weakest precondition with respect to $\langle+W\rangle$ is thus

$$
\beta \Leftrightarrow(f \vee h) \wedge(f \vee \neg h) \wedge(\neg f \vee h),
$$

and the weakest precondition with respect to $\langle-W\rangle$ is the trivial formula true, since the positive literal $W$ does not appear in the CNF representation of $\alpha^{*}$. In other words, if $W$ is deleted, then $\alpha^{*}$ will always be satisfied in the resulting database state.

Ordered with respect to $f, \alpha^{*}$ is equivalent to

$$
(f \vee \neg W \vee h) \wedge(f \vee \neg W \vee \neg h) \wedge(\neg f \vee \neg W \vee h),
$$

and the weakest precondition of $\alpha^{*}$ with respect to $\langle-f\rangle$ is

$$
\begin{aligned}
\beta & \Leftrightarrow(\neg W \vee h) \wedge(\neg W \vee \neg h) \\
& \Leftrightarrow \neg W .
\end{aligned}
$$

Meanwhile, the weakest precondition of $\alpha^{*}$ with respect to $\langle+f\rangle$ is

$$
\beta \Leftrightarrow \neg W \vee h,
$$

which is logically implied by the constraint $\alpha^{*}$. This means that if $\alpha^{*}$ is satisfied in the current database state, then it will also be satisfied in the state resulting from the execution of $\langle+f\rangle$. We make this notion precise in the next section with the definition of threatening and assisting updates. 
Similarly, ordered with respect to $h, \alpha^{*}$ is equivalent to

$$
(h \vee \neg W \vee f) \wedge(h \vee \neg W \vee \neg f) \wedge(\neg h \vee \neg W \vee f)
$$

The weakest precondition of $\alpha^{*}$ with respect to $\langle-h\rangle$ is

$$
\begin{aligned}
\beta & \Leftrightarrow(\neg W \vee f) \wedge(\neg W \vee \neg f) \\
& \Leftrightarrow \neg W,
\end{aligned}
$$

and the weakest precondition of $\alpha^{*}$ with respect to $\langle+h\rangle$ is

$\neg W \vee f$,

which is also logically implied by $\alpha^{*}$.

In summary, three of the six updates described, $\langle-W\rangle,\langle+f\rangle$, and $<$ $+h>$, have a weakest precondition that is logically implied by the constraint $\alpha^{*}$. This means that if $\alpha^{*}$ is satisfied in the current database state, then it will also be satisfied in the state resulting from the execution of any of these three updates. This is not the case for the other updates, $\langle+W\rangle$, $<-f\rangle$, and $\langle-h\rangle$. In the notation of the following section, we say that the updates $\langle+W\rangle,\langle-f\rangle$, and $\langle-h\rangle$ threaten the constraint $\alpha^{*}$, while the others do not. In fact, for each of the other three updates, it is possible to move from a state in which $\alpha^{*}$ is not satisfied to one in which it is; in this context we say that $\langle-W\rangle,\langle+f\rangle$, and $\langle+h\rangle$ assist $\alpha^{*}$. In the next section we define these terms and, more importantly, provide an algorithm for determining which updates threaten or assist a given constraint.

\section{THREATENING AND ASSISTING UPDATES}

In the maintenance of integrity constraints, we assume that the database state is consistent prior to the update; i.e. it satisfies all integrity constraints. Thus, the primary focus is on updates which threaten integrity constraints. However, another relevant class of updates is those which assist integrity constraints by restoring consistency in a database where the constraint is not currently satisfied. We now formally define these notions. We refer to $S$ as the set of all database states and to $I$ as an element of $S$.

Definition 3 Let $M$ be an event and $\alpha$ a static formula.

1. $M$ threatens $\alpha$ if and only if $\exists I \in S$ such that $I \vDash \alpha$ and $I \vDash \neg(M \alpha)$;

2. $M$ assists $\alpha$ if and only if $\exists I \in S$ such that $I \models \neg \alpha$ and $I \vDash M \alpha$. 
Given an atomic update $\langle+\tau\rangle$ or $\langle-\tau\rangle$, where $\tau$ is a positive literal, we express each integrity constraint in which $\tau$ appears in reduced conjunctive normal form, ordered with respect to $\tau$ :

$$
\alpha \Leftrightarrow C_{1} \wedge \cdots \wedge C_{k} \wedge C_{k+1} \wedge \cdots \wedge C_{m}
$$

where each clause $C_{i}$ is of the form $P_{i 1} \vee P_{i 2} \vee \cdots \vee P_{i n}$,

$$
P_{i 1} \Leftrightarrow\left\{\begin{array}{cc}
\tau & \text { if } i \leq k ; \text { or } \\
\neg \tau & \text { if } i>k
\end{array},\right.
$$

each $P_{i j}$ is either $P_{j}$ or $\neg P_{j}$, and $\left\{\tau, P_{2}, \ldots, P_{n}\right\}$ is a set of distinct positive literals.

Definition 4 We say that two clauses $C_{i}$ and $C_{j}$ of (the reduced $C N F$ form of) $\alpha$ match if $C_{i}$ is of the form

$$
\tau \vee P_{i 2} \vee \cdots \vee P_{i n}
$$

and $C_{j}$ is of the form

$$
(\neg \tau) \vee P_{i 2} \vee \cdots \vee P_{\text {in }}
$$

Notice that it is not possible for every clause in $\alpha$ to have a matching clause, since then $\alpha$ would be of the form

$$
\begin{aligned}
&\left(\tau \vee P_{12} \vee \cdots \vee P_{1 n}\right) \wedge \cdots \wedge\left(\tau \vee P_{k 2} \vee \cdots \vee P_{k n}\right) \wedge \\
&\left(\neg \tau \vee P_{12} \vee \cdots \vee P_{1 n}\right) \wedge \cdots \wedge\left(\neg \tau \vee P_{k 2} \vee \cdots \vee P_{k n}\right) \\
& \Leftrightarrow\left(P_{12} \vee \cdots \vee P_{1 n}\right) \wedge \cdots \wedge\left(P_{k 2} \vee \cdots \vee P_{k n}\right) .
\end{aligned}
$$

The latter formula is in conjunctive normal form with respect to a proper subset of $\left\{\tau, P_{2}, \ldots, P_{n}\right\}$, which contradicts the assumption that the original conjunctive normal form of $\alpha$ was reduced.

The following theorems show how to decide whether an atomic update threatens an integrity constraint. The proof of the first theorem is given in the appendix; the proof of the second is entirely analogous.

Theorem 3 Let $\tau$ be a positive literal, and

$$
\alpha \Leftrightarrow C_{1} \wedge \cdots \wedge C_{k} \wedge C_{k+1} \wedge \cdots \wedge C_{m}
$$

a static formula in conjunctive normal form, ordered with respect to $\tau$ as above. Then the following statements are equivalent: 
1. $\langle+\tau>$ threatens $\alpha$;

2. $\langle-\tau>$ assists $\alpha$;

3. There is at least one clause $C_{i}$ with $i>k$ that does not have a matching clause.

Theorem 4 Let $\tau$ be a positive literal, and

$\alpha \Leftrightarrow C_{1} \wedge \cdots \wedge C_{k} \wedge C_{k+1} \wedge \cdots \wedge C_{m}$

a static formula in conjunctive normal form, ordered with respect to $\tau$ as above. Then the following statements are equivalent:

1. $\langle-\tau\rangle$ threatens $\alpha$;

2. $\langle+\tau\rangle$ assists $\alpha$;

3. There is at least one clause $C_{i}$ with $i \leq k$ that does not have a matching clause.

The third condition in Theorems 3 and 4 provides an efficient method for determining precisely which updates threaten which constraints. The equivalence of the first and third conditions guarantees that a complete list of all threatening updates can be generated. Furthermore, the dual equivalence of threatening insertions and assisting deletions, and vice versa, will be utilised in Section 5 to restore the satisfaction of constraints using the Action/Condition/ Correction (ACC) rule.

Example 2 We continue our discussion of the constraint

$$
\alpha^{*}: W \rightarrow f \wedge h
$$

in Example 1 of section 3. With respect to $W, \alpha^{*}$ is equivalent to

$$
(\neg W \vee f \vee h) \wedge(\neg W \vee f \vee \neg h) \wedge(\neg W \vee \neg f \vee h)
$$

In this case $k=0$ since $W$ does not appear as a positive literal; consequently, $\langle+W\rangle$ threatens $\alpha^{*}$ since there exist three clauses $C_{i}, i>0$ without a matching clause. Meanwhile, $\langle-W\rangle$ does not threaten $\alpha^{*}$ since there are no clauses $C_{i}$ with $i \leq 0$.

With respect to $f, \alpha^{*}$ is equivalent to

$$
(f \vee \neg W \vee h) \wedge(f \vee \neg W \vee \neg h) \wedge(\neg f \vee \neg W \vee h)
$$

Here $k=2$ and the first and third clauses match. Thus, $\langle+f\rangle$ does not threaten $\alpha^{*}$ since $C_{3}$ has a matching clause, and $\langle-f\rangle$ does threaten $\alpha^{*}$ since $C_{2}$ does not have a matching clause. 
Similarly, with respect to $h, \alpha^{*}$ is equivalent to

$$
(h \vee \neg W \vee f) \wedge(h \vee \neg W \vee \neg f) \wedge(\neg h \vee \neg W \vee f)
$$

$k=2,\langle+h\rangle$ does not threaten $\alpha^{*}$, while $<-h>$ does threaten $\alpha^{*}$ since $C_{2}$ has no matching clause.

Using Theorems 3 and 4 , the preceding analysis also shows that the updates that assist $\alpha^{*}$ are $\langle+W\rangle,\langle-f\rangle$ and $\langle-h\rangle$. We will discuss the implementation of assisting updates in the context of the corrections in the Action/Condition/Correction (ACC) rule of section 5.

\section{UPDATE RULES}

In this section two rule types are introduced for supporting the design of correct update semantics. The rule types correspond to the following natural interpretations for integrity maintenance: (1) a rule type that tests whether the update will violate the integrity of a constraint before the update is executed, and (2) a rule type that executes the update, and then performs corrective action if necessary to maintain integrity. Rollback of an update is viewed here as a form of corrective action. In the first type, the Condition/Action (CA) rule, the condition must be true in order for the update to execute; otherwise it is not executed. In the second type, the Action/Condition/Correction (ACC) rule, the update executes, and if the condition is true then corrective action is executed to restore a consistent database. Given an integrity constraint and an update which could result in this constraint being violated, the derivation procedure automatically derives the condition in the CA rule and the condition and corrective action in the ACC rule. The formal basis in EFL is a significant advantage for using these rules analyses rather than other rule approaches, such as ECA(McCarthy et al. 1989). The logic-theoretic semantics provided by EFL permit straightforward correctness proofs shown later in this section.

\subsection{Condition/Action (CA) Rule}

The CA rule focuses on the updates which threaten an integrity constraint. From the previous analysis for an arbitrary constraint, we have the weakest precondition with respect to each threatening update. This fulfills the necessary parameter for the CA rule.

Definition 5 Given a static formula $\beta$ that is equivalent to the weakest pre- 
condition of an integrity constraint $\alpha$ with respect to an atomic update $M$ which threatens $\alpha$, the corresponding Condition/Action (CA) rule is given by

\section{$<$ if $\beta$ then $M$ fi $>$.}

The CA rule is an event in EFL and thus can participate in subsequent analyses. Since $\beta$ is the weakest precondition, if the specified condition is true, then a database resulting from the execution of $M$ satisfies $\alpha$.

\section{Example 3 Let}

$$
\alpha^{*}: W \rightarrow(f \wedge h)
$$

be the constraint studied in the previous section, and let $\langle+W\rangle$ be the proposed update which threatens $\alpha^{*}$. The weakest precondition of $\alpha^{*}$ with respect to $\langle+W\rangle$ is

$$
(f \vee h) \wedge(f \vee \neg h) \wedge(\neg f \vee h)
$$

so the CA rule for this update would be

$$
\text { if }(f \vee h) \wedge(f \vee \neg h) \wedge(\neg f \vee h) \text { then }<+W>
$$

\subsection{Action/Condition/Correction (ACC) Rule}

In the ACC rule we focus on the set of atomic updates that assist $\alpha^{*}$ as well. The trigger in the $\mathrm{AC}$ rule is a threatening update. The Condition is derived also from the weakest precondition as in the CA rule. Those updates that assist $\alpha^{*}$ are the source for the corrective actions in the ACC rule. Since $\beta$ is complete in the sense that it expresses all clauses which must be satisfied for the constraint not to be violated after the update executes, $\neg \beta$ is used to imply corrective action is necessary. The disjunctive normal form of $\neg \beta$ supplies a disjunct of mutually exclusive clauses, each signaling the need for corrective action. The structure of each clause, i.e. the conjuncted literals, determines the specific candidate updates for corrective action, from the set of assisting updates.

Definition 6 Let $M$ be an atomic update that threatens a constraint $\alpha$, and let $\beta$ be the weakest precondition for $\alpha$ with respect to $M$. The corresponding Action/Condition/Correction (ACC) rule is given by 


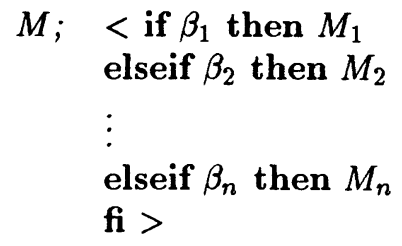

where

1. $\neg \beta \Leftrightarrow \beta_{1} \vee \beta_{2} \vee \ldots \vee \beta_{n}$ (disjunctive normal form);

2. $M_{1}, M_{2}, \ldots, M_{n}$ are sequences of updates that assist $\alpha$; and

3. for all $i, 1 \leq i \leq n$, either $\beta_{i} \Rightarrow M_{i} \beta$ or $M_{i}$ undoes $M$.

In other words, each event $M_{i}$ either restores the satisfaction of $\beta$, or undoes the update $M$. In the former case, this also restores the constraint $\alpha$, since $\beta$ is a necessary and sufficient condition for $\alpha$ to hold after the update executes. In the latter case, if $M=\langle+\tau\rangle$, then the corrective update $M_{i}=\langle-\tau\rangle$ undoes $M$ and restores the original database state, in which $\alpha$ was satisfied.

Example 4 Once again, we consider the constraint $\alpha^{*}$ and the threatening update $\langle+W\rangle$. The disjunctive normal form of the negation of $\beta$ is given by

$$
\neg \beta \Leftrightarrow(\neg f \wedge \neg h) \vee(\neg f \wedge h) \vee(f \wedge \neg h)
$$

The disjunctive clauses then provide the conditions for an ACC rule template. The updates $M_{i}$ are chosen from among the updates that assist $\alpha^{*}$. Suppose, for example, that the designer wishes to model the following corrective action: if a frame does not exist for a window assembly WA, then the desired update $<+W>$ should be undone, or "rolled back" by subsequently executing the correction $\langle-W\rangle$. If, however, a frame does exist for WA, but the corresponding has_part relation $h$ is missing, then the correction should consist of inserting the missing relation $h$. Then $M_{1}$ and $M_{2}$ should be $\langle-W\rangle$, and $M_{3}$ should be $<+h>$, giving the following ACC rule.

$$
\begin{aligned}
<+W>; & <\text { if }(\neg f \wedge \neg h) \text { then }<-W> \\
& \text { elseif }(\neg f \wedge h) \text { then }<-W> \\
& \text { elseif }(f \wedge \neg h) \text { then }<+h> \\
& \text { fi }>
\end{aligned}
$$

In many cases the designer anticipates certain states in which the constraint is violated and does want a complete rule template, i.e. one that has all the elseif components. This is the designer's prerogative; the analysis can provide an accurate and complete rule specification, but the designer's extra domain knowledge is required to reduce the number of tests after the update, to edit 
the corrective actions, or to substitute a rollback of the threatening update in place of the corrective actions supplied by the analysis.

\subsection{Soundness of the CA and ACC Rules}

The following theorem asserts the soundness of the CA and ACC rules as tools for update semantic analysis.

Theorem 5 Let $\alpha$ be a formula, $M$ an update that threatens $\alpha$, and let $\beta$ be the static formula equivalent to the weakest precondition of $\alpha$ with respect to $M$. Then $\alpha \Rightarrow M_{C A} \alpha$ and $\alpha \Rightarrow M_{A C C} \alpha$, where $M_{C A}$ is a $C A$ rule of the form

$$
<\text { if } \beta \text { then } M \text { fi }>\text {, }
$$

and $M_{A C C}$ is an $A C C$ rule of the form

$$
\begin{aligned}
M ; & <\text { if } \beta_{1} \text { then } M_{1} \\
& \text { elseif } \beta_{2} \text { then } M_{2} \\
& \vdots \\
& \text { elseif } \beta_{n} \text { then } M_{n} \\
& \text { fi }>
\end{aligned}
$$

Proof. First we show that $\alpha \Rightarrow M_{C A} \alpha$. Let $I$ be a state such that $I \vDash \alpha$. If $I \vDash \beta$ then $I \vDash M \alpha$ since $\beta \Rightarrow M \alpha$ by the definition of the weakest precondition, and $I \vDash M_{C A} \alpha$. If $I \vDash \neg \beta$, then $I \vDash M_{C A} \alpha$ because $I \vDash \alpha$.

Now we show that $\alpha \Rightarrow M_{A C C} \alpha$. Again, let $I$ be a state such that $I \vDash \alpha$. If $I \vDash \beta$ then $I \vDash \neg \beta_{i}$ for all $I$, so $I \vDash M_{A C C} \alpha$ if and only if $I \vDash M \alpha$, which follows from the fact that $\beta \Rightarrow M \alpha$ and $I \vDash \beta$. If $I \vDash \neg \beta$, then $I \vDash \beta_{i}$ for exactly one $i, 1 \leq i \leq n$, and there are two cases for the corrective action $M_{i}$. If $M_{i}$ undoes $M$, then $I \vDash M_{A C C} \alpha$ because $I \vDash M ; M_{i} \alpha$ if and only if $I \vDash \alpha$. If $\beta_{i} \Rightarrow M_{i} \beta$, then $M_{i}$ must insert or delete one of the literals in the discatenation $\beta$, and not the literal inserted or deleted by $M$. Thus the order of the two updates is irrelevant and $I \vDash M_{A C C} \alpha$ if and only if $I \vDash\left(M_{i} ; M\right) \alpha$ if and only if $I \vDash M_{i}(M \alpha)$ if and only if $I \vDash M_{i} \beta$, which follows from the fact that $\beta_{i} \Rightarrow M_{i} \beta$. 


\section{IMPLEMENTATION ISSUES}

\subsection{User/System-Supplied Data}

One implementation problem is that update rules as described do not provide a mechanism to identify or create new objects. An object identified in a relationship to be inserted may not exist in the database, thus violating a constraint. For example, if an attempt (possibly as the result of a rule propagation) is being made to insert a Connect relationship between a frame and a hinge, and the frame does not already exist, then an existence constraint between the relationship and its participating objects is violated. A front-end selection mechanism gives the user the opportunity to identify the object at run time in order to enforce the integrity constraint. An alternative approach is to have the system generate an object automatically, which is then added to the database as a persistent object and as a component in the relationship. Both mechanisms should be provided in order to give the schema designer a full range of behavioral choices.

These mechanisms are modeled here as the meta-events Select $(f)$ and Generate $(f)$. Their operational semantics is outside the formal system, but are viewed here simply as providers of objects bound to the variable $f$. Select $(f)$ abstracts the interaction process of prompting a user for a frame object; Generate $(f)$ abstracts the system process of automatically constructing a frame object. The meta-events do not affect the rule generation process. Designer intervention is needed to distinguish among cases where Select, Generate, or neither is appropriate.

The following example illustrates the incorporation of these events in update rules.

$$
\begin{aligned}
\text { Objects: } & \text { Frame }(F), \text { Hinge }(H) \\
\text { Relationship: } & \text { Connect }(F, H) \\
\text { Integrity Constraint: } & \text { if Connect }(F, H) \text { then } \operatorname{Frame}(F)
\end{aligned}
$$

Rules 1, 2, and 3 below are ACC rules corresponding to three variations of enforcing this constraint with respect to an insert of the relationship, Connect between a frame and a hinge; rule 1 denies the insert if the frame does not exist; rule 2 prompts the user to supply a frame; and rule 3 generates a frame automatically.

Rule1: Rule2:

$$
\begin{aligned}
& <+\operatorname{Connect}\left(F_{1}, H_{1}\right)>\text {; } \\
& <\text { if } \neg \operatorname{Frame}\left(F_{1}\right) \text { then }<-\operatorname{Connect}\left(F_{1}, H_{1}\right)>\text { fi }>
\end{aligned}
$$

$$
\begin{aligned}
& <+ \text { Connect }\left(F_{1}, H_{1}\right) ; \\
& <\text { if } \neg \text { Frame }\left(F_{1}\right) \text { then } \\
& \quad<-\operatorname{Connect}\left(F_{1}, H_{1}\right)>\text {; } \\
& \quad<\operatorname{Select}\left(F_{2}\right)>;
\end{aligned}
$$


Rule3 :

$$
\text { fi }><+ \text { Connect }\left(F_{2}, H_{1}\right)>
$$

$$
\begin{aligned}
& <+ \text { Connect }\left(F_{1}, H_{1}\right) ; \\
& <\text { if } \neg \text { Frame }\left(F_{1}\right) \text { then } \\
& \quad<- \text { Connect }\left(F_{1}, H_{1}\right) ; \\
& \quad<\text { Generate }\left(F_{2}\right)>; \\
& \quad<+ \text { Connect }\left(F_{2}, H_{1}\right)> \\
& \text { fi }>\quad
\end{aligned}
$$

\subsection{Evaluable Queries}

At run time conditions in the update rules are evaluated, returning a true or false answer. Care must be taken to ensure that these conditions can be evaluated as reasonable queries (Van Gelder 1991). Reasonable queries are characterised by the following properties which support an efficient evaluation strategy: (1) The solutions of reasonable queries can be completely characterised by the constants in the database and the query itself; i.e. domain independence (Fagin 1982, Ullman 1988). (2) Efficient mechanisms exist to decide whether a query is domain independent or not, to translate a domain independent query into a form for evaluation such as the relational algebra, and to execute the query. The class of evaluable formulas (Demolombe 1982, Van Gelder 1991) has been identified as the largest subset of domain independent formulas satisfying these properties. An example of an evaluable formula is $f(x) \Leftrightarrow \neg p(x) \wedge q(x) ; x$ is restricted to be a constant in the relation $q$. On the other hand, $g(x, y) \Leftrightarrow p(x) \vee q(y)$ is not an evaluable formula since $g(x, y)$ is true for arbitrary $y$ when $p(x)$ is true, and vice versa.

The automatic generation of update rules may result in conditions not in this class. Therefore the weakest precondition $\beta$ is restricted to members of this class. A similar restriction is imposed on the maintainable integrity constraints in related work.

\subsection{Method Complexity}

The derivation process of update rules for a given integrity constraint can be realised in cubic polynomial time as a function of the number of operators in a formula. This section sketches the algorithm and the respective complexities for each of its steps. The algorithm is presented primarily to demonstrate the effectiveness of the overall process.

Let $\alpha$ denote the integrity constraint to be analysed, and let $N$ denote the number of operators in $\alpha$. 
1. Standard transformation of $\alpha$ into reduced CNF: $O\left(N^{3}\right)$.

2. Finding the Weakest Precondition: $O\left(\log _{2} N\right)$. Given $\alpha$ in reduced-CNF and an atomic update $M$, find the weakest precondition $\beta$ of $\alpha$. This step requires a search of $\alpha_{b i n}$ to select the appropriate subtree as $\beta$.

3. Construct Threatens $(\alpha)$ and $\operatorname{Assists}(\alpha): O\left(N^{3}\right)$. Given a database in which $\alpha$ is satisfied, Threatens $(\alpha)$ is the set of atomic updates whose execution may result in $\alpha$ not holding. In the following steps, let $\tau$ denote an atomic formula in $\alpha$.

Repeat the following steps until all distinct atomic formulas in $\alpha$ have been considered, with the notation as in Lemma 1 of section 3 and Definition 4 of section 4. (1) Order the clauses in $\alpha$ by $\tau$. (2) Determine the matched clauses in $\alpha$. (3) If there is an unmatched clause, $C_{i}$, with $i>k$ then add $\langle+\tau\rangle$ to Threatens $(\alpha)$ and $\langle-\tau\rangle$ to $\operatorname{Assists}(\alpha)$; otherwise take no action. (4) If there is an unmatched clause, $C_{i}$, with $i \leq k$ then add $\langle-\tau\rangle$ to Threatens $(\alpha)$ and $\langle+\tau\rangle$ to $\operatorname{Assists}(\alpha)$; otherwise take no action.

4. Generate Update Rules: $O(N)$.

(a) CA Rule: Fill in the rule template with $\beta$ and $M$ respectively, $O(1)$.

(b) ACC Rule: This is a two step task to generate a set of rules from which the database designer can choose, $O(N)$, following the template of Definition 6 of section 5.2. (1) Construct the disjunctive normal form of $\neg \beta$. (2) Fill in the condition templates with the disjunctive clauses of $\neg \beta$. (3) For each disjunctive clause of $\neg \beta$, assign a subset of updates from Assists $(\alpha)$ as follows: for each positive literal $\tau$ in $\beta_{i}$, add $\langle-\tau\rangle$ to $m_{i}$, and for each literal $\neg \tau$ in $\beta_{i}$, add $\langle+\tau\rangle$ to $m_{i}$. At this point designer feedback is required to construct $M_{i}$, the sequence of corrective actions in the rule template, from $m_{i}$, or to elect to undo the triggering update.

\section{CONCLUSION}

Integrity constraints are very useful in advanced database systems such as those found in the CAD domain. Constraints express in declarative form the complex semantics of relationships among objects. In most advanced systems, update rules are specified to enforce the integrity constraints. A system which can generate update rules both correctly and automatically is a very important component for such advanced applications. In this paper, we describe a method for generating update rules from integrity constraints in first-order logic which can be proven to generate

1. rules guaranteed to be correct

2. a complete list of actions which can invalidate a constraint

3. a complete list of actions which can restore consistency. 
This is a result of our formal framework, which provides a uniform semantics for integrity constraints and update rules. Our method is an efficient syntactic derivation process. In addition, it provides rule templates for specifying whether the integrity constraint is tested before or after an update is executed.

This research is part of a larger project to develop a design environment for architectural databases, in which both static and behavioral semantics are part of the schema specification language. An important part of this project will be a rule generator, based on the methods described here, as well as related rule analysis tools to be developed.

\section{REFERENCES}

A. Aiken, J. Widom, and J. Hellerstein, "Behavior of Database Production Rules: Termination, Confluence, and Observable Determinism", Proc. 1992 SIGMOD, SIGMOD Record, 21(2), 59-68.

J. Brawner and J. Vorbach, "An Algorithmic Logic Approach to Formalizing Database Update Semantics," to appear in the J. of Applied NonClassical Logics, 1998.

S. Ceri, P. Fraternali, S. Paraboschi, and L. Tanca, "Automatic Generation of Production Rules for Integrity Maintenance," ACM Trans. on Database Sys., 19(3), 367-422, 1994.

S. Ceri and J. Widom, "Deriving Production Rules for Constraint Maintenance," Proc. 16th VLDB Conf., 566-577, 1990.

R. Demolombe, "Syntactical Characterization of a Subset of Domain Independent Formulas," Tech. Report, ONERA-CERT, 1982.

R. Fagin, "Horn Clauses and Database Dependencies," J. ACM 29(4), 952$985,1982$.

P. Fraternali and S. Paraboschi, "A Review of Repairing Techniques for Integrity Maintenance," Rules in Databas Systems 1993, 333-346.

G. Kramer, "Using Degrees of Freedom Analysis to Solve Geometric Constraint Systems," ACM SIGGRAPH, 371-378, June 1991.

D.R. McCarthy and U. Dayal, "The Architecture of An Active Data Base Management System", Proceedings of the 1989 ACM SIGMOD International Conference on the Management of Data, 215-224.

G. Mirkowska and A. Salwicki, Algorithmic Logic, D. Reidel, 1986.

S.H. Mullins and D.C. Anderson, "A Positioning Algorithm for Mechanical Assemblies with Closed Kinematic Chains in Three Dimensions", Second Symposium on Solid Modeling and Applications, 1993, 271-280.

J.M. Nicolas and K. Yazdanian, "Integrity Checking in Deductive Databases," in Logic and Databases, (H. Gallaire and J. Minker, eds.) Plenum (New York), 1978, 325-244.

X. Qian, "Synthesizing Database Transactions," Proc. 16th VLDB Conf., 552$564,1990$.

J. Peckham and B. MacKellar and M. Doherty, "A Data Model for the Ex- 
tensible Support of Explicit Relationships in Design Databases,", The Very Large Database Journal, April, 1995, 157-192.

K.D. Schewe, B. Thalheim, J. W. Schmidt, and I. Wetzel, "Integrity Enforcement in Object-Oriented Databases," Proc. 4th Int. Workshop on Foundations of Models and Languages for Data and Objects, Oct. 1992, Volkse, Germany, 181-206.

J. Ullman, Principles of Database and Knowledge-base Systems, Vol. 1, Computer Science Press, 1988.

S. Urban and L. Delcambre, "Constraint Analysis: A Design Process for Specifying Operations on Objects," IEEE Trans. Knowl. and Data Eng., 2(4), 391-400, 1990.

S. Urban and M. Desiderio, "CONTEXT: A CONsTraint EXplanation Tool," Data \& Knowl. Eng., 8, 153-183, 1992.

S. Urban, A. Karadimce, and R. Nannapaneni, "The Implementation and Evaluation of Integrity Maintenance Rules in an Object-Oriented Database," Proc. 8th Int'l. Conf. on Data Eng., 565-572, 1992.

A. Van Gelder and R. Topor, "Safety and Translation of Relational Calculus Queries," ACM Trans. on Database Sys., 16(2), 367-422, 1991.

B. Wüthrich, "On Updates and Inconsistency Repairing in Knowledge Bases," Proc. 9th IEEE Int. Conference on Data Engineering, Vienna, Austria, Apr. 1993, 608-615.

\section{BIOGRAPHIES}

Jim Brawner received his B.A. degree in English and Mathematical Sciences from Williams College in 1985, and his Ph.D. in Mathematics from the University of North Carolina at Chapel Hill in 1992, under the direction of Jonathan Wahl. His research interests include algebraic curves and surfaces, updates in database systems, and the use of technology in the classroom. After teaching at St. John's University in Jamaica, New York, he is currently on the faculty of Armstrong Atlantic State University, where his non-academic interests include knitting, juggling, and word puzzles. e-mail: James_Brawner@mailgate.Armstrong.edu

Bonnie MacKellar is an associate professor of computer science at Western Connecticut State University. Previously, from 1989-1992, she was employed as an assistant professor at New Jersey Institue of Technology. Her research interests include active database systems, intelligent CAD systems, object-oriented data modeling, and computers in the building industry. She received her B.S. degree from Boston University, U.S.A., and her M.S. and PhD degrees in computer science from the University of Connecticut, U.S.A., in 1986 and 1989 respectively. email: bonnie@theodora.wcsu.ctstateu.edu

Joan Peckham has an M.S. in mathematics (1975), an M.S. in Computer Science (1985) and a PhD in Computer Science (1990) from the University of Connecticut. She is currrently an associate professor of Computer 
Science at the University of Rhode Island. Her research interests include active and CAD database systems, and object-oriented design and analysis. e-mail:joan@cs.uri.edu

James Vorbach is an Associate Professor in the Department of Mathematics and Computer Science at St. John's University. He is the director of the Innovation Lab (I-LAB), an initiative to provide undergraduate computer science students with concrete design experiences. His research interests include information systems design, database logic, and semantic modeling. He received his Ph.D. in Applied Mathematical Sciences at the University of Rhode Island. e-mail: vorbachj@stjohns.edu.

\section{A. APPENDIX}

\section{A.1. Event-Formula Language (EFL)}

\section{A.1.1. Language}

The alphabet for the language $L$ includes:

1. an enumerable set $U$ of constants;

2. an enumerable set $V$ of variables;

3. a finite set $T$ of unary relation symbols corresponding to types in a specification;

4. a finite set $\Delta$ of binary relation symbols corresponding to relationships in a specification;

5. the standard logical symbols for equality $(=)$, connectives $(\vee, \wedge, \neg)$ and quantifiers $(\forall, \exists)$.

There are three kinds of well-formed expressions: terms, formulas, and events. A term is a variable or a constant. An atomic formula is an expression of the form $\tau\left(t_{1}\right)$ or $\delta\left(t_{1}, t_{2}\right)$, where $t_{1}$ and $t_{2}$ are terms, $\tau \in T$, and $\delta \in \Delta$. The set $F_{0}$ of static formulas is the least set containing the atomic formulas suchthat if $\alpha, \beta \in F_{0}$ and $x$ is a variable then $(\alpha \vee \beta),(\neg \alpha)$ and $(\exists x) \alpha$ belong to $F_{0}$. We use the standard logical equivalences of propositional logic; e.g. we abbreviate $\neg(\neg \alpha \vee \neg \beta)$ to $\alpha \wedge \beta, \neg \alpha \vee \beta$ to $\alpha \rightarrow \beta,(\alpha \rightarrow \beta) \wedge(\beta \rightarrow \alpha)$ to $\alpha \leftrightarrow \beta$, and $\neg(\exists x)(\neg \alpha)$ to $(\forall x) \alpha$, and $\alpha \vee(\neg \alpha)$ to true.

The set $\Pi$ of events is the least set such that if $t_{1}$ and $t_{2}$ are terms, $\tau \in T$, $\delta \in \Delta, \alpha \in F_{0}$ and $M_{1}$ and $M_{2}$ are events then

1. the identity event $I d$ is an event;

2. the atomic updates $+\tau\left(t_{1}\right),-\tau\left(t_{1}\right),+\delta\left(t_{1}, t_{2}\right)$ and $-\delta\left(t_{1}, t_{2}\right)$ are events; and

3. (if $\alpha$ then $M_{1}$ else $M_{2}$ fi) and $\left(M_{1} ; M_{2}\right)$ are events. 
We abbreviate (if $\alpha$ then $M$ else $I d$ fi) to (if $\alpha$ then $M$ fi) and (if $\alpha_{1}$ then $M_{1}$ else (if $\alpha_{2}$ then $M_{1}$ fi) fi) to (if $\alpha_{1}$ then $M_{1}$ elseif $\alpha_{2}$ then $M_{2}$ fi).

The set of all formulas is the least extension of the set $F_{0}$ of static formulas such that

1. if $\alpha$ is a formula and $M$ is an event then $M \alpha$ is a formula;

2. if $\alpha$ and $\beta$ are formulas and $x$ is a variable then $(\alpha \vee \beta),(\neg \alpha)$ and $(\exists x) \alpha$ are formulas.

If a formula is not static, it is said to be dynamic.

A database schema is a triple $\langle L, A, E\rangle$, where $A$ is a nonempty finite set of formulas and $E$ is a nonempty finite set of events in the given language $L$.

\section{A.1.2. Semantics}

The Herbrand universe of the language $L$ is the set $U$ of its constant symbols. The Herbrand base $B$ of $L$ is the set of all ground atomic formulas $\tau\left(c_{1}\right)$, $\delta\left(c_{1}, c_{2}\right)$, where $c_{1}, c_{2} \in U$. Any subset of the Herbrand base is identified with a database state. A data structure $D$ for $L$ is a pair $\left(S, m_{d}\right)$, where $S=2^{B}$ is the set of all states and $m_{d}$ is a dynamic meaning function on ground events that is a binary relation on the set of states.

We denote by $v: U \cup V \rightarrow U$ a valuation map from the set of terms to the set of constants. Given a valuation $v$, a variable $x$ and a term $t$, we denote by $v_{x}^{t}$ the valuation obtained from $v$ by substituting the term $t$ for the variable $x$; i.e. $v_{x}^{t}(x)=v(t)$, and $v_{x}^{t}(y)=v(y)$ for all variables $y \neq x$. We define equality of terms in the usual manner: two terms $t_{1}$ and $t_{2}$ are equal under the valuation $v$, written $\left(t_{1}=t_{2}\right)[v]$, if and only if

$$
v\left(t_{1}\right)={ }_{U} v\left(t_{2}\right)
$$

where $=_{U}$ is the standard notion of equality on the set $U$.

For our purposes we define the notions of satisfaction and dynamic meaning as follows. If $D=\left(S, m_{d}\right)$ is a data structure, $I \in S$ is a database state, and $v$ is a valuation, we define the satisfaction of a formula $\alpha$ in the state $I$ under $v$, denoted $I \vDash \alpha[v]$, and the dynamic meaning of an event $M$ under $v$, denoted $m_{d}(M[v])$, by simultaneous induction as follows.

1. If $\alpha$ is an atomic formula then $I \models \alpha[v]$ if and only if $\alpha[v] \in I$.

2. If $\alpha$ and $\beta$ are formulas then $I \vDash(\alpha \vee \beta)[v]$ if and only if either $I \vDash \alpha[v]$ or $I \vDash \beta[v]$.

3. If $\alpha$ is a formula then $I \vDash(\neg \alpha)[v]$ iff it is not the case that $I \vDash \alpha[v]$.

4. If $\alpha$ is a formula and $x$ is a variable then $I \vDash(\exists x) \alpha[v]$ iff there is a constant $c \in U$ such that $I \vDash \alpha\left[v_{x}^{t}\right]$.

5. The dynamic meaning of the identity event is $m_{d}(I d)=\{(I, I): I \in S\}$. 
6. If $\alpha$ is an atomic formula then

$$
\begin{aligned}
& m_{d}(+\alpha[v])=\{(I, J): J=I \cup \alpha[v]\} \text { and } \\
& m_{d}(-\alpha[v])=\{(I, J): J=I \backslash \alpha[v]\}
\end{aligned}
$$

7. If $M_{1}$ and $M_{2}$ are events then $m_{d}\left(\left(M_{1} ; M_{2}\right)[v]\right)=$

$$
\left\{(I, J): \exists K \in S \text { such that }(I, K) \in m_{d}\left(M_{1}[v]\right) \text { and }(K, J) \in m_{d}\left(M_{2}[v]\right)\right\}
$$

8. If $\alpha$ is a static formula and $M_{1}$ and $M_{2}$ are events then

$$
m_{d}\left(\left(\text { if } \alpha \text { then } M_{1} \text { else } M_{2} \text { fi) }[v]\right)=\right.
$$

$$
\begin{aligned}
& \left\{(I, J): I \models \alpha[v] \text { and }(I, J) \in m_{d}\left(M_{1}[v]\right)\right\} \cup \\
& \left\{(I, J): I \models(\neg \alpha)[v] \text { and }(I, J) \in m_{d}\left(M_{2}[v]\right)\right\}
\end{aligned}
$$

9. If $\alpha$ is a formula and $M$ is an event then $I \models(M \alpha)[v]$ if and only if

$\exists J \in S$ such that $(I, J) \in m_{d}(M[v])$ and $J \models \alpha[v]$.

We emphasise that the above definitions are made with respect to a particular data structure $D=\left(S, m_{d}\right)$. If there is some ambiguity over which data structure is involved, we write $I \vDash \models_{D} \alpha[v]$. The notions of validity, tautology and equivalence are defined with respect to the above concepts.

A formula $\alpha$ is valid in a state $I$, denoted $I \vDash \alpha$, if and only if $I \vDash \alpha[v]$ for every valuation $v$. A formula $\alpha$ is valid for a data structure $D=\left(S, m_{d}\right)$, denoted $\vDash_{D} \alpha$, if and only if $I \vDash_{D} \alpha$ for every state $I \in S$. A formula $\alpha$ is a tautology, denoted $\vDash \alpha$, if and only if $\models_{D} \alpha$ for every data structure $D$ in the language $L$. We define the equivalence of two formulas in the standard way: $\alpha$ is equivalent to $\beta$, denoted $\alpha \Leftrightarrow \beta$, if and only if $\alpha \leftrightarrow \beta$ is a tautology. Similarly, $\alpha$ implies $\beta$, denoted $\alpha \Rightarrow \beta$, if and only if $\alpha \rightarrow \beta$ is a tautology.

Notice that a formula is valid in a state $I$ if and only if it is satisfied in $I$ under all possible valuations. There is no corresponding notion of validity for events since satisfaction of events is not defined. The dynamic meaning of an event is only defined with respect to a valuation.

\section{A.2. Proof of Theorem 2}

We give the proof for the case where $M$ is an atomic deletion $<-\tau\rangle$. The other case is entirely analogous. First we show that $\beta \rightarrow M \alpha$ is valid. Let $I \in S$ be any database state for which $I \vDash \beta$. If $J$ is any state for which 
$(I, J) \in m_{d}(M)$ then $J=I \backslash\{\tau\}$. Since $\tau$ is not an atomic formula of $\beta$, $J \vDash \beta$ as well, and of course $J \vDash(\neg \tau)$. Since $\alpha \wedge(\neg \tau)$ is equivalent to $\beta \wedge(\neg \tau)$ then $J \vDash \alpha \wedge(\neg \tau)$, so $J \vDash \alpha$ and $I \vDash M \alpha$.

If $\gamma$ is any formula such that $\vDash(\gamma \rightarrow M \alpha)$ then let $I$ be any database state for which $I \vDash \gamma$. From the assumption, $I \vDash M \alpha$ and $J \vDash \alpha$ for some $J$ such that $(I, J) \in m_{d}(M)$; i.e., for $J=I \backslash\{\tau\}$. Therefore, $J \vDash \alpha \wedge(\neg \tau)$ and $J \vDash \beta \wedge(\neg \tau)$. Since $\tau$ is not an atomic formula of $\beta, I \vDash \beta$.

\section{A.3. Proof of Theorem 3}

(1) $\Leftrightarrow(2):\langle+\tau\rangle$ threatens $\alpha$ if and only if $\exists I \in S$ such that $I \vDash \alpha$ and $I \vDash \neg(<+\tau\rangle \alpha)$ if and only if $\left.\exists(I, J) \in m_{d}(<+\tau\rangle\right)$ such that $I \vDash \alpha$ and $J \vDash \neg \alpha$ if and only if $\exists I, J \in S$ such that $J=I \cup\{\tau\}, I=J \backslash\{\tau\}, I \vDash \alpha$ and $J \vDash \neg \alpha$ if and only if $\exists(J, I) \in m_{d}(<-\tau>)$ such that $J \vDash \neg \alpha$ and $I \vDash \alpha$ if and only if $\exists J \in S$ such that $J \vDash \neg \alpha$ and $J \vDash<-\tau>\alpha$ if and only if $<-\tau>$ assists $\alpha$.

(1) $\Rightarrow(3)$ : Suppose every clause $C_{i}$ with $i>k$ has a matching clause. Then for all $i>k$,

$$
\begin{aligned}
\alpha & \Rightarrow\left(\tau \vee P_{i 2} \vee \cdots \vee P_{i n}\right) \wedge\left(\neg \tau \vee P_{i 2} \vee \cdots \vee P_{i n}\right) \\
& \Leftrightarrow P_{i 2} \vee \cdots \vee P_{i n}
\end{aligned}
$$

and $\alpha \Rightarrow \beta$, where $\beta$ is the discatenation of $\alpha$ with respect to $\langle+\tau\rangle$. Since $\beta$ is the weakest precondition by Theorem 2 , then $\beta \Rightarrow<+\tau>\alpha$. Hence, $\alpha \Rightarrow\langle+\tau\rangle \alpha$, and $\langle+\tau\rangle$ does not threaten $\alpha$.

$(3) \Rightarrow(1)$ : Let $C_{i}$ be a clause without a matching clause, where $C_{i}$ is of the form

$\neg \tau \vee P_{i 2} \vee \cdots \vee P_{i n}$.

Let $I$ be any database state in which the clause

$$
\gamma=\neg \tau \wedge \neg P_{i 2} \wedge \cdots \wedge \neg P_{i n}
$$

is satisfied; that is, $I \vDash \gamma$. Since $C_{i}$ does not have a matching clause, then for all $j \leq k$ there is at least one literal $P_{j l}$ such that $P_{j l} \Leftrightarrow \neg P_{i l}$, so that

$$
\gamma \Rightarrow \neg P_{i l} \Leftrightarrow P_{j l} \Rightarrow C_{j}
$$

for all $j \leq k$. Since $\gamma \Rightarrow \neg \tau$ as well, then $\gamma \Rightarrow \alpha$, and $I \vDash \alpha$. On the other hand, if $J$ is any state such that $\left.(I, J) \in m_{d}(<+\tau\rangle\right)$ then $J=I \cup\{\tau\}$ and $\mathbf{J}$ $\vDash \tau \wedge \neg P_{i 2} \wedge \cdots \wedge \neg P_{\text {in }}$, and $\mathrm{J} \vDash \neg C_{i}$, and $\mathrm{J} \vDash \neg \alpha$, and $\mathrm{I} \vDash \neg<+\tau>\alpha$, so $\langle+\tau>$ threatens $\alpha$. 